\title{
FISIOTERAPIA
}

\section{Características hemodinámicas y terapéuticas de pacientes con diagnóstico de Debilidad Adquirida en la Unidad de Cuidados Intensivos (DAUCI), en el Sanatorio San Lucas, 2014-2017}

\author{
Martín Inmediato Ghetti ${ }^{1,2}$
}

\begin{abstract}
Resumen
Introducción: La tasa de pacientes que ingresan a $\mathrm{UCl}$ y desarrollan DAUCl es elevada. A esto se suman los escasos protocolos presentes en el abordaje de la DAUCI. La evidencia publicada sobre la efectividad de la fisioterapia temprana en esta área es muy limitada.
\end{abstract}

Objetivo: El objeto principal del estudio consistió en identificar las características hemodinámicas y terapéuticas de los pacientes con diagnóstico de debilidad adquirida en unidad de cuidados intensivos, en el Sanatorio San Lucas, desde año 2014 al 2017.

Material y Método: El presente estudio es de diseño descriptivo, no experimental, retrospectivo, transversal de enfoque cuantitativo. Los sujetos de estudios fueron 20 fichas de pacientes ingresados a la unidad de cuidados intensivos con diagnóstico de $\mathrm{DAUCl}$. Se empleó una ficha de recolección de datos con las variables del estudio, en donde se procedió a registrar los datos conformes a lo establecido, los datos fueron tabulados y procesados en EPIDAT 4.1 y Microsoft Excel 2016.

Resultados: La distribución mayoritaria hacia el sexo femenino, puede deberse a la prevalencia de la afectación en mujeres debido a las diferencias morfológicas presentes, las cuales las vuelven más susceptibles al desarrollo de DAUCl, de la misma forma, estudios recientes mencionan que efectivamente el sexo femenino es el predominante en cuanto al desarrollo de la condición. La variabilidad presente en distribución de la edad, que puede referirse a la diversidad presente en la unidad de cuidados intensivos, aunque se observa la mayor distribución en la franja etaria de 68 y 82 años, la prevalencia de la DAUCl se observa en pacientes adultos mayores, quienes tienen mayor probabilidad de desarrollar comorbimortalidades. Uno de los datos de mayor relevancia del

1. Universidad Iberoamericana, Paraguay.

2. Universidad del Norte, Paraguay.

E-mail: martin.inmediato@hotmail.com

DOI: $10.26885 /$ rcei.foro.2019.225

Trabajo publicado en acceso abierto bajo Licencia Creative Commons. 
estudio fue la alteración de los valores de la PiMáx constatándose una relevante disminución de los valores con lo fisiológicamente recomendado para cada sujeto de estudio. El estudio refiere que un $30 \%$ de los sujetos de estudio, tuvo como Diagnóstico de Ingreso Dengue o Sx. Febril de Dengue. Últimos estudios acerca del Dengue, indican que, los mismos producen secuelas a nivel del sistema nervioso, como ser diferentes tipos de neuropatías, no obstante, a nivel mundial no se encuentra la relación entre el Dengue y la DAUCl, una de las condicionantes del estudio consiste en que nacionalmente el Dengue es una endemia.

Se observa una hiperoxemia relacionada por exceso de oxigenoterapia, la relación existente entre hiperoxemia y DAUCl, no está evidenciada en estudios recientes, no obstante, si existe una relación entre oxigenoterapia excesiva frente a la aparición de neuropatías, asimismo se puede evidenciar en los estudios que la DAUCl es una condición multifactorial.

Conclusiones: Las características hemodinámicas de los pacientes al ingreso en la Unidad de cuidados intensivos se mantuvieron fluctuantes, la mayoría de los sujetos de estudio se encontraban con valores fuera del rango fisiológico normal. Al egreso los valores se normalizaron en gran parte, dejando escasos sujetos con alteraciones en las variables. La terapéutica kinésica puede orientar a una falta de registro y de criterios al momento de la aplicación de las diferentes técnicas kinésicas.

Palabras clave: $\mathrm{DAUCl}$, terapéutica, Pimáx.

\section{Referencias}

Connolly, B., Salisbury, L., O’Neill, B., Geneen, L., Douiri, A., Grocott, M., Hart, N., Walsh, T., Blackwood, B. (2015). Exercise rehabilitation following intensive care unit discharge for recovery from critical illness. Cochrane Database of Systematic Reviews, 6. 10.1002/14651858.CD008632.pub2

Dargains, N., Bratos, A., et al. (2016). Debilidad adquirida en la unidad cuidados intensivos incidencia y factores de riesgo. Conference: $26^{\circ}$ Congreso Argentino de terapia intensiva.

Diaz, L., Dargains, N., Inchaustegui, J., Bratos, A., Milagros, M., Ardariz, C., et al. (2017). Weakness acquired in the intensive care unit. Incidence, risk factors and their association with inspiratory weakness. Observational cohort study. Rev Bras Ter Intensiva, 29(4), 466-75.

Martí, J. (2016). Debilidad muscular adquirida en la unidad de cuidados intensivos: ¿un problema con una única solución? Enfermería Intensiva, 27, 41-43

Mejía, A., Martínez, N., Nieto, O., Camacho, M., Tomas, E., Martínez, B. (2018). Movilización Temprana Como Prevención Y Tratamiento Para La Debilidad Adquirida En La Unidad De Cuidados Intensivos En Pacientes En Ventilación Mecánica. Experiencia En Un Hospital De Segundo Nivel. Eur Sci J, 14(21), 19-30. http://eujournal.org/index.php/esj/article/ view/11037/10610 